\title{
Performance of RIPASA and AIR Scores in a Middle- Eastern Subset of Patients with Suspected Diagnosis of Acute Appendicitis
}

\section{Mohammed Faisal}

Suez Canal University Faculty of Medicine Ahmed Bayoumi

Suez Canal University Faculty of Medicine

Ahmed Abo Bakr

Suez Canal University Faculty of Medicine

\section{Marcus Edlehamre}

Linkoping University Faculty of Medicine: Linkopings universitet Medicinska Fakulteten

Islam Khaled ( $\nabla$ dr.islam.ahmed@med.suez.edu.eg )

Suez Canal University Faculty of Medicine https://orcid.org/0000-0001-6577-4312

\section{Research}

Keywords: Acute appendicitis, RIPASA, AIR, histopathology

Posted Date: October 23rd, 2020

DOl: https://doi.org/10.21203/rs.3.rs-93856/v1

License: (9) This work is licensed under a Creative Commons Attribution 4.0 International License. Read Full License 


\section{Abstract}

Background: Early and definitive acute appendicitis (AA) diagnosis remains a great challenge in clinical practice worldwide. Currently, several risk scores are available for the clinical assessment of AA, although there are differences in their accuracy rates. The aim of the study was to assess and analyze the accuracy of the acute inflammatory response (AIR) and Raja Isteri Pengiran Anak Saleha Appendicitis (RIPASA) scores in an Egyptian population.

Methods: A series of 310 patients, $61 \%$ male with a median age of 18 years, with Rt. lliac fossa pain were clinically evaluated for suspicion of AA. All patients were assessed using both the AIR and RIPASA scores. Following evaluation, all patients underwent appendectomy. A final diagnosis of AA was based on histopathology. Two predetermined cutoff values for the AIR score and one cutoff value for the RIPASA score was evaluated for sensitivity, specificity, and accuracy. Statistical analysis was performed using SPSS version 22. Cross tabulation was performed to assess sensitivity, specificity, positive and negative predictive values along with other tests for parametric and non-parametric variables.

Results: The sensitivities of the RIPASA score cutoff value of $\geq 7.5$ and AIR score cutoff values of $>4$ and $>8$ were $83.8 \%, 92.2 \%$, and $33.1 \%$, accordingly, while the specificities were $69.2 \%, 76.7 \%$, and $84.6 \%$, respectively. AIR score of $>4(91 \%)$ was more accurate compared to AIR score of $>8(37.4 \%)$ and RIPASA score of $\geq 7.5(82.5 \%)$.

Conclusion: An AIR score of $>4$ had better sensitivity, specificity, and accuracy compared to a RIPASA score of $\geq 7.5$ for the Egyptian population. However, an AIR score of $>8$ was more specific. Therefore, an evaluation of patients in low-income countries using one of these scoring systems is highly recommended to avoids unnecessary hospitalization along in negative individuals, in addition to preventing complications in positive cases.

\section{Background}

Appendicitis is one of the most frequent acute surgical disorders with one in seven life-long prevalence (1). The incidence of acute appendicitis (AA) has been steadily declining since the late 1940s. In developed countries, AA affects approximately 5,750 per 100000 population per year in developing countries, with the highest incidence between 10 and 30 years of age $(2,3)$.

Geographical differences have been asserted, with a long-life risk for AA of 9,8 and \% in the USA, Europe, and Africa, respectively (4). There is also great variation in the presentation, severity, radiological evaluation, and surgical management of patients with AA in relation to the average income in each country (5).

Clinical diagnosis is a craft taking time to understand. It is based on information gleaned from a large number of variables in the patient's history, clinical examination, and laboratory studies. Experience with many patientgs is required to understand the variations in this complex pattern of information. However, 
even experienced surgeons often err by giving too much credence to pain and tenderness and too little to the inflammatory response (6).

The diagnosis of AA is primarily clinical, and only contrast enhanced computed tomography (CT) has a high enough sensitivity and specificity to establish the correct diagnosis $(7,8)$. However, high cost and limited availability are often factors that hinder its utility especially in developing countries.

A clinical scoring system that has acceptable sensitivity and specificity with a low rate of appendectomy would be especially useful. The Acute Inflammatory Response (AIR) and Raja Isteri Pengiran Anak Saleha Appendicitis (RIPASA) scoring systems have been recently developed to aid in the rapid diagnosis of AA especially in developed countries to replace the remote Alvarado score that has been used in Western nations $(9,10)$. We prospectively compared RIPASA with AIR scores in patients presenting with right iliac fossa (RIF) pain with suspected appendicitis in our institution.

\section{Methods}

A prospective analysis was performed on 310 patients presenting with signs and symptoms suggestive of AA from September 2017 to April 2018. Written consent was obtained from each patient before enrollment in the study. The current analysis was carried out at Suez Canal University Hospitals, Ismailia, Egypt. All patients were evaluated using RIPASA (9) and AIR scores (10) by a general surgery registrar, and they were monitored by an external observer (one of the authors) who is familiar with the parameters of both scores to avoid bias and under- or overestimation. All patients presented to the emergency room (ER) with right iliac fossa pain, which raise an overall suspicion in AA, both males and females $\geq 16$ years of age were included. Patients with the following conditions were excluded: $<16$ years or $\geq 60$ years of age, $\mathrm{BMI} \geq 40 \mathrm{~kg} / \mathrm{m}^{2}$, presence of inflammatory bowel disease, a pathological diagnosis of malignant appendicular lesions, appendicular perforation, pregnancy, and patients with a RIF mass or a history of urolithiasis.

A complete history, including the nature and duration of symptoms, was taken from each patient. All patients received a complete physical examination by the registrar in the ER. Blood work-up included hemoglobin level, white leucocyte count (WBC), and C-reactive protein (CRP). A urine pregnancy test for females in the reproductive age group who presented with amenorrhea was performed. A routine urinalysis with microscopy and pelvi-abdominal ultrasound was the investigation of choice in female patients who have been complaining of a suspicious gynecological disease that could not be excluded by history and clinical examination in order to avoid inclusion of complicated ovarian cysts, polycystic ovary and other pure gynecological lesions in the current study. After the previous parameters for patients' final enrollment had set, the ineligible patients were excluded from the study (Fig. 1). All patients were subjected to the following:

1. RIPASA and AIR scoring for each clinically diagnosed case of appendicitis. 
2. Histopathological confirmation was the gold standard confirmation after appendectomy and assessment was done considering positive or negative specimen.

\section{Statistical analysis}

All analyses were performed using SPSS version 22.0 (IBM, Armonk, New York, U.S) and GraphPad Prism version 8.0.1 (GraphPad Software, San Diego, California, U.S). A p value $<0.05$ was considered significant.

Variables were tested for their pattern of distribution using the Kolmogorov-Smirnov test and visual assessment of histograms. Data with normal distribution were expressed as mean \pm standard deviation while non-normally distributed variables were presented as median and interquartile range (IQR). Following histopathological examination, all patients were classified into 2 groups based on the presence of absence of acute appendicitis. To assess possible differences between the groups, for parametric and non-parametric variables we used the unpaired student $t$ and Mann Whitney $U$ tests, respectively. To compare categorical variables, the Chi-square test was used. Cross tabulation was performed to assess sensitivity, specificity, positive, and negative predictive values (PPV and NPV), respectively. The diagnostic accuracy of each scoring system was calculated using histopathology as the gold standard for diagnostic confirmation. To detect the area under the curve (AUC) for each score, a receiver operating characteristic (ROC) curve was plotted to evaluate both scores using SPSS version 22.0 software.

\section{Results}

Following the exclusion of 44 patients, 310 were identified for final analysis, with the majority being males $(61 \%)$ and a male: female ratio of 1.6:1 (Table 1). The final diagnosis was based on histopathological findings following open appendectomy. A total of $92 \%$ of patients had histologically proven appendicitis, while $8 \%$ had a normal (negative) appendix. The median age among all patients was 18 years with no difference between those with and without appendicitis (Table 1). 
Table 1

Baseline data among all patients:

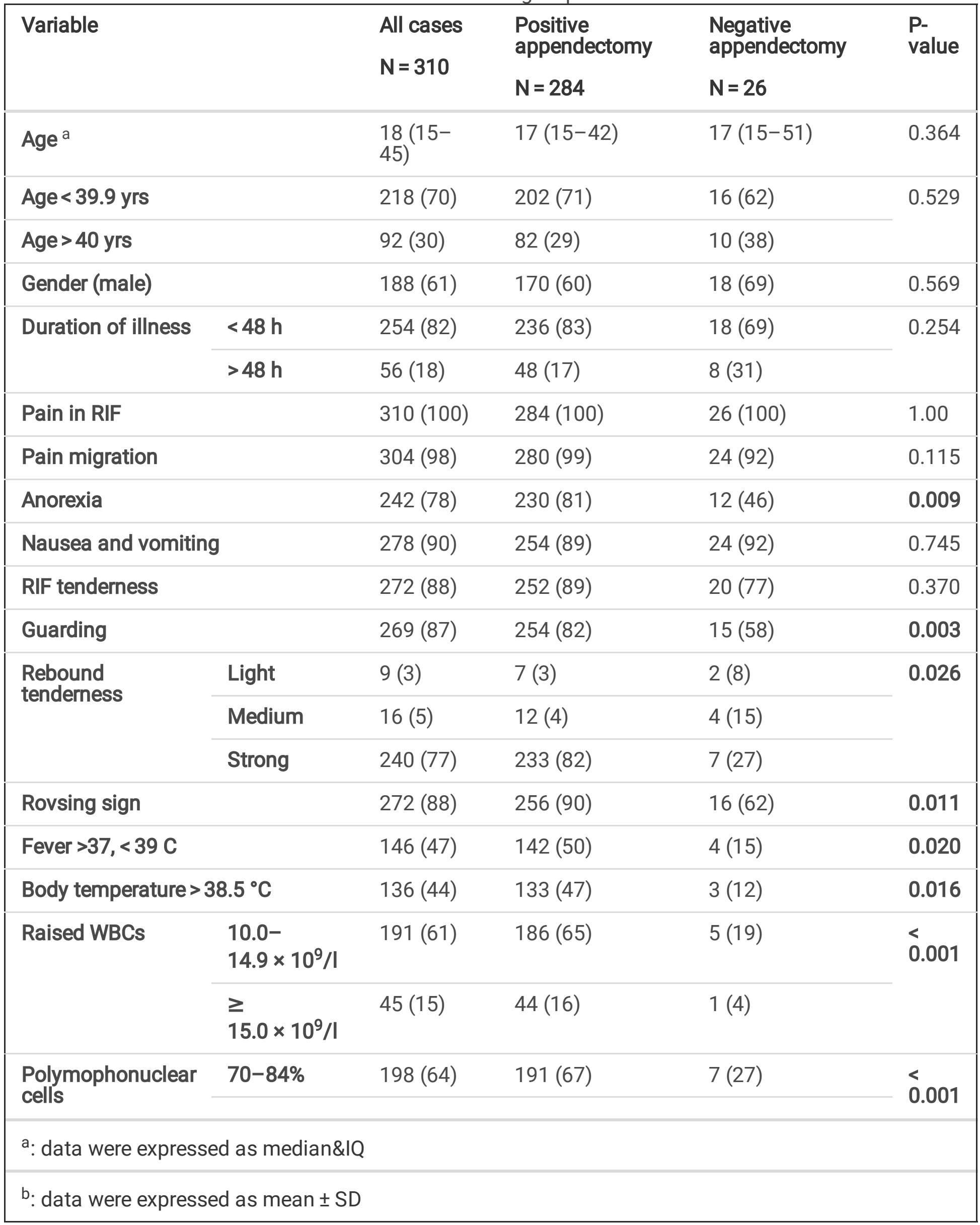




\begin{tabular}{|c|c|c|c|c|c|}
\hline \multirow[t]{2}{*}{ Variable } & & All cases & Positive & Negative & \multirow{2}{*}{$\begin{array}{l}\mathrm{P} \text { - } \\
\text { value }\end{array}$} \\
\hline & & $N=310$ & $N=284$ & $N=26$ & \\
\hline & $\geq 85 \%$ & $38(12)$ & $34(12)$ & $4(15)$ & \\
\hline \multirow[t]{2}{*}{ CRP concentration } & $10-49 \mathrm{~g} / \mathrm{l}$ & $178(57)$ & $167(59)$ & $11(42)$ & \multirow[t]{2}{*}{0.043} \\
\hline & $\geq 50 \mathrm{~g} / \mathrm{l}$ & $24(8)$ & $14(5)$ & $10(38)$ & \\
\hline Urine analysis (-ve) & & $240(77)$ & $232(82)$ & $8(31)$ & $\hat{0} .001$ \\
\hline \multirow[t]{2}{*}{ RIPASA score ${ }^{a}$} & median\&IQ & $\begin{array}{l}12.5(9.5- \\
13.5)\end{array}$ & $13(10.5-13.5)$ & $7(7-10)$ & \multirow[t]{2}{*}{$<.001$} \\
\hline & Range & $5-15$ & $5-15$ & $6-11$ & \\
\hline \multirow[t]{2}{*}{ AIR score $^{a}$} & median\&IQ & $8(6-9)$ & $8(6-9)$ & $4(3.5-5.5)$ & \multirow{2}{*}{$\begin{array}{l}<.001 \\
0.001\end{array}$} \\
\hline & Range & $3-12$ & $3-12$ & $3-10$ & \\
\hline \multirow[t]{2}{*}{$\operatorname{LOS}^{b}$} & mean $\pm S D$ & $3.45 \pm 1.78$ & $3.53 \pm 1.79$ & $3.23 \pm 1.53$ & \multirow[t]{2}{*}{0.678} \\
\hline & Range & $1-12$ & $1-12$ & $1-6$ & \\
\hline \multicolumn{6}{|c|}{ a. data were expressed as median\&IQ } \\
\hline b. data were express & as mean $\pm s$ & & & & \\
\hline
\end{tabular}

Most patients had an onset of symptoms less than 48 hours prior to surgery. All patients suffered from pain in the RIF (100\%), with nausea and vomiting in $90 \%$, RIF tenderness in $88 \%$, and rebound tenderness in $77 \%$. These were all statistically significant in the positive subgroup (Table 1).

Only $47 \%$ of the patients presented with fever, Rovsing sign was positive in $88 \%$, WBCs were elevated in $61 \%, \mathrm{CRP}>50 \mathrm{~g} / \mathrm{L}$ was present in $8 \%$, and a negative urinalysis was present in $77 \%$. A significantly greater proportion of patients presented with these signs and laboratory findings in the positive compared to the negative group (Table 1).

The RIPASA and AIR scores for all patients had a median of 12.5 (range 5-15) and 8 (range 6-9), respectively. There were higher median scores in the positive compared to the negative group (Table 1).

Although there was a wide range of hospital stay (1-12 days) in the positive group, their mean length of stay was not significantly different than the negative group (Table 1 ).

Based on the histopathological final diagnosis, a RIPASA score with a cutoff value $\geq 7.5$ was capable of detecting 238 out of 284 truly positive cases and correctly excluding 18 of 26 truly negative with a sensitivity and specificity of $83.80 \%$ and $69.23 \%$, respectively (Tables 2 and 4 ). However, an AIR score with a cutoff value $>4$ achieved better results, as it could identify 262 out of 284 truly positive cases and 
correctly exclude 20 of 26 truly negative cases with a sensitivity and specificity of $92.25 \%$ and $76.92 \%$, respectively (Tables 3 and 4). An AIR score with a cutoff value $>8$ had a higher specificity but lower sensitivity, $84.61 \%$ and $33.09 \%$ respectively (Table 3).

Table 2

shows relationship between RIPASA score and the histopathological diagnosis

\begin{tabular}{|llll|}
\hline & Positive cases & Negative cases & Total \\
\hline RIPASA score $\geq 7.5$ & 238 & 8 & 246 \\
\hline RIPASA score $<7.5$ & 46 & 18 & 64 \\
\hline Total & 284 & 26 & 310 \\
\hline
\end{tabular}

Table 3

shows relationship between RIPASA score and the histopathological diagnosis

\begin{tabular}{|llll|}
\hline & Positive cases & Negative cases & Total \\
\hline AIR score $>4$ & 262 & 6 & 268 \\
\hline AIR score $\leq 4$ & 22 & 20 & 42 \\
\hline Total & 284 & 26 & 310 \\
& Positive cases & Negative cases & Total \\
\hline AIR score $>8$ & 94 & 4 & 98 \\
\hline AIR score $\leq \mathbf{8}$ & 190 & 22 & 212 \\
\hline Total & 284 & 26 & 310 \\
\hline
\end{tabular}


Table 4

shows sensitivity, specificity, PPV and NPV of both AIR and RIPASA scores:

\begin{tabular}{|c|c|c|c|c|c|c|}
\hline \multirow{3}{*}{ Sensitivity } & \multirow[t]{2}{*}{ AIR $>4$} & \multirow{2}{*}{\multicolumn{2}{|c|}{$(95 \% \mathrm{Cl})$}} & \multicolumn{3}{|c|}{ RIPASA $\geq 7.5$} \\
\hline & & & & $(95 \% \mathrm{Cl})$ & & $(95 \% \mathrm{Cl})$ \\
\hline & $92.25 \%$ & $\begin{array}{l}86.23- \\
95.87\end{array}$ & $33.09 \%$ & $\begin{array}{l}25.57- \\
41.55\end{array}$ & $83.80 \%$ & $\begin{array}{l}76.47- \\
89.24\end{array}$ \\
\hline Specificity & $76.92 \%$ & $\begin{array}{l}45.98- \\
93.83\end{array}$ & $84.61 \%$ & $\begin{array}{l}53.66- \\
97.28\end{array}$ & $69.23 \%$ & $\begin{array}{l}38.88- \\
89.64\end{array}$ \\
\hline PPV & $97.76 \%$ & $\begin{array}{l}93.09- \\
99.42\end{array}$ & $95.91 \%$ & $\begin{array}{l}84.86- \\
99.28\end{array}$ & $96.74 \%$ & $\begin{array}{l}91.38- \\
98.95\end{array}$ \\
\hline NPV & $47.61 \%$ & $\begin{array}{l}26.38- \\
69.66\end{array}$ & $10.37 \%$ & $5.54-18.19$ & $28.12 \%$ & $\begin{array}{l}14.39- \\
46.97\end{array}$ \\
\hline $\begin{array}{l}\text { Positive likelihood } \\
\text { ratio }\end{array}$ & 3.99 & $1.48-10.79$ & 2.15 & $0.58-7.86$ & 2.72 & $1.20-6.17$ \\
\hline $\begin{array}{l}\text { Negative likelihood } \\
\text { ratio }\end{array}$ & 0.10 & $0.05-0.18$ & 0.79 & $0.68-0.91$ & 0.23 & $0.15-0.36$ \\
\hline Pre-test probability & 10.9 & & 10.9 & & 10.9 & \\
\hline Post-test probability & 43.4 & & 23.32 & & 29.6 & \\
\hline Accuracy & $91 \%$ & & $37.4 \%$ & & $82.5 \%$ & \\
\hline
\end{tabular}

An AIR score $>4$ is more diagnostic than a RIPASA score $\geq 7.5$ (PPV, 97.7\% vs. 96.7; NPV, $47.6 \%$ vs. $28.1 \%$, respectively) and highly accurate (91\% vs. $82.5 \%$, respectively) (Table 4). The AUC was 0.827 and 0.846 for AIR and RIPASA scores, respectively, (Fig. 2).

The rate of postoperative complications was approximately $9 \%$, A total of 9 patients developed chest infection (2.9\%) and 2 developed venous thromboembolism (0.6\%). Three of the 9 also had infections at the surgical site, and 2 of 9 developed a pelvic abscess. Infections at the surgical site were detected in 11 patients $(3.5 \%)$, while a pelvic abscess was identified in $8(2.6 \%)$. Four patients $(1.6 \%)$ had isolated urinary tract infections following surgery (Fig. 3).

\section{Discussion}

Acute appendicitis is one of the most popular emergencies in surgery around the world. The gold standard treatment is appendectomy. However, a debate now exists regarding the possibility of medically treating AA (11). A delay in diagnosis may lead to complications including perforation, abscess formation, peritonitis, and even death (12). However, an increased rate of surgery for normal appendices has undesirable consequences such as post-operative adhesions and ileus (11). These drawbacks to aggressive surgery have led to the use of ultrasonography and $\mathrm{CT}$ to reduce the rate of negative appendectomies and improve diagnostic accuracy. However, these additional diagnostic modalities have 
led to increased financial burdens and health care costs particularly in less developed countries with limited resources (13).

Given these findings and the difficulties of over and underestimation in the diagnosis of $A A$, several scoring systems have been developed. Since its introduction in 1986, the Alvarado scoring system has been the most popular (14). Subsequently, modified Alvarado scoring systems have emerged to improve the original Alvarado score's lower specificity and sensitivity when applied in western nations (15). During the past decade, a new scoring system was developed to treat deficiencies in the Alvarado and modified Alvarado scoring systems in Asian populations. The RIPASA scoring system was initiated in 2010, and it depends solely on history, clinical examination, and 2 blood tests $(9,16)$. The AIR score was initiated parallel to the RIPASA score to overcome the drawbacks of the previous scores. This score incorporates two simple lab tests (WBC count and CRP) in its scheme. The AIR score assesses the intensity of AA in relation to an elevated WBC count together with the percent of segmented neutrophils and CRP(10).

The most common presenting symptom in our study was RIF pain which was present in in almost all cases. A Brazilian study by Von-Muehlenn et al., to compare AIR against the Alvarado score in 147 patients, found RIF pain in 140 patients (95.3\%). They reported vomiting in $51.7 \%$ and a temperature > $38.5^{\circ} \mathrm{C}$ in $27.9 \%$, while $90 \%$ of our patients had nausea and vomiting and $44 \%$ had a temperature $>38.5^{\circ}$ C (17). Shuaib et al., in a Middle Eastern study, found the following incidences of clinical symptoms: pain in the right iliac fossa, 99.3\%; anorexia, 58.1\%; nausea and vomiting, 81\%; and fever, 39\% (13).

The total negative appendectomy rate in the current study was $8.4 \%$, which is less than the rates documented by Shuaib et al., (13), Chong et al., (17) and Rathod et al., (18) who documented rates of $18.4 \%, 22.9 \%$, and $20.69 \%$, respectively.

ROC analysis was performed in the present study to ascertain a cut off score for RIPASA with higher sensitivity and specificity by plotting the true positive rate against the false positive rate for different cut points of a RIPASA score. The AUC for the RIPASA system was greater than that of the AIR (Fig. 2). However, when the AIR score was calculated for a score $>8$, there was a higher specificity but much lower sensitivity.

To the best of our knowledge, only a few studies have directly compared the RIPASA and AIR scoring systems for the diagnosis of AA. In the present study, the sensitivity of an AIR score $>4$ was greater than that of a RIPASA score $\geq 7.5$ and significantly better than AIR scores $>8$. The sensitivities for an AIR score $>4$, an AIR score $>8$, and a RIPASA score $\geq 7.5$ was $92.25 \%, 33.09 \%$, and $83.8 \%$, respectively. The specificity of an AIR score $>8$ was greater than an AIR score $>4$ and a RIPASA score $\geq 7.5,84.6 \%, 76.9 \%$ and $69.2 \%$, respectively.

The present study illustrates that the negative appendectomy rate (Table 2 and 3 ) was recorded in AIR score $>4$ in $2.2 \%$ ( 6 out of 268) patients subjected to the score and in RIPASA $\geq 7.5$ score, it was recorded in $2.9 \%$ (8 out of 246 ) of cases, while the AIR score $>8$ demonstrates a negative appendectomy rate of 
$4 \%$ of cases. The highest rate of suspected patients who proved later to be truly positive by histopathogy was registered in AIR > 4 score with $97.8 \%$ (262 out of 268 ).

Table 5 illustrates a comparison between the current study and previous studies $(16,19)$ which evaluated the RIPASA versus the AIR scoring system in the diagnosis of AA. The highest sensitivity and specificity, 93.1\% and $91.6 \%$, respectively, were recorded by Karami et al(16). However, Bolívar-Rodríguez et al., in a study performed in Mexico, reported a sensitivity of $97.2 \%$ and a specificity of $27.6 \%$ (19). These findings show that a RIPASA score with a cut-off $>7.5$ can successfully diagnose AA with a greater than $80 \%$ accuracy and higher PPV and positive likelihood ratio than 2 of the 3 previously compared studies.

Other studies have compared the RIPASA score to different scoring systems. Shauib et al. (13) reported that a RIPASA score $\geq 7.5$ yielded acceptable diagnostic accuracy measures except for a NPV of $78.5 \%$. They found the sensitivity, specificity, PPV, and diagnostic accuracy to be $94.5 \%, 88.0 \%, 97.2 \%$, and $93.38 \%$, respectively. Chong et al. (17) reported sensitivity, specificity, PPV, NPV and diagnostic accuracy rates of $98.0 \%, 81.3 \%, 85.3 \%, 97.4 \%$, and $91.8 \%$, respectively, using a score $>7.5$. Using the RIPASA score, Rathod et al., (18) determined sensitivity, specificity, PPV, NPV, and diagnostic accuracy of $82.61 \%$, $88.89 \%, 96.61 \%, 57.14 \%$, and $83.91 \%$, respectively.

Andersson was the first to implement the AIR scoring system for the diagnosis of AA in 2008. That report noted a sensitivity of $96 \%$ and a specificity of $73 \%$ with a cutoff threshold $>4$ and a sensitivity and specificity of $37 \%$ and $99 \%$, respectively when the cutoff point was $>8(10)$. In the present study, the AIR score cutoff was examined at two different values, $>4$ and $>8$. A cutoff point $>4$ yielded a sensitivity, specificity, PPV, NPV, and diagnostic accuracy of $92.25 \%, 76.9 \%, 96.7 \%, 47.7 \%$, and $91 \%$, respectively. However, a cutoff point $>8$ yielded disappointing results with poor sensitivity, NPV, and accuracy, meaning it would be difficult to exclude truly negative individuals.

A prior study by Karami et al. (16) demonstrated that a RIPASA score cutoff $>8$ yielded a sensitivity, specificity, PPV, NPV, and diagnostic accuracy of 78.4\%, 91.6\%, 98.5\%, and $36.6 \%$ respectively. Based on our current study, an AIR score $>4$ results in the highest diagnosis accuracy for AA of $91 \%$ due to its combined abilities to detect truly positive cases in addition to excluding truly negative patients who experience right lower abdominal pain.

In another comparative study similar to ours, Scott et al. found high sensitivities for intermediate and high risk patients with appendicitis (90\%), and for patients with advanced appendicitis (98\%), when the AIR score was > 5 (20). De Castro et al. noted a 93\% sensitivity and $85 \%$ specificity for an AIR $>4(21)$, while in the current study, the sensitivity and specificity for an AIR $>4$ were $92.2 \%$ and $76.9 \%$, respectively. This contrasts to a $33 \%$ sensitivity and $84.6 \%$ specificity with a score set at $>8$.

We postulate that both a RIPASA score $\geq 7.5$ and an AIR $>4$ have a very good ability to detect truly sick patients when they are at high risk. The current era of scoring systems aiding in the preoperative diagnosis of appendicitis could help to avoid unnecessary laparotomies. The current study adds more precision to the validity of both AIR and RIPASA as objective tools that can be applied to our population 
to support decision-making in the management of a patient with suspected AA. Our observed rate of negative appendectomies, 26 out of 310 patients (8.4\%), is certainly within an acceptable range.

The RIPASA and AIR scoring systems did not show a significant difference in the diagnosis of AA in an Egyptian population. They both contain easily obtained parameters from the history, physical examination, and a few simple blood tests. Both scoring systems are beneficial for making a quick decision in resource limited and low-middle income countries without the need for imaging assistance.

Managing patients with suspected AA remains a challenge and the recommended management approach is indeed unknown, even after ultrasound, CT, and diagnostic laparoscopy were introduced.

\section{Conclusion}

An AIR score of $>4$ had better sensitivity, specificity, and accuracy compared to a RIPASA score of $\geq 7.5$; however, an AIR score of $>8$ was more specific for the Egyptian population. Both scoring systems could safely prevent hospitalization and unnecessary investigations in patients with doubtful diagnosis. Additional randomized, controlled trials are needed to validate the best scoring system for acute appendicitis diagnosis.

\section{List Of Abbreviation}




\begin{tabular}{|ll|}
\hline - AA & Acute Appendicitis \\
\hline - AIR & Acute Inflammatory Response \\
\hline RIPASA & Raja Isteri Pengiran Anak Saleha Appendicitis \\
\hline RIF & Right lliac Fossa \\
\hline BMI & Body Mass Index. \\
\hline CRP & Emergency Room. \\
- WBC & White Leucocyte Count \\
- IQR & Interquartile Range \\
\hline PPV & positive, and negative predictive values \\
- NPV & Negative Predictive Values \\
\hline CRP & C-reactive protein \\
\hline AUC & Area Under the Curve \\
\hline ROC & Receiver Operating Characteristic \\
\hline CT & Computerized Tomography \\
\hline
\end{tabular}

\section{Declarations}

\section{Ethical approval and consent to participate}

All procedures performed in our study involving human participants was approved by the ethical committee of Suez Canal University Hospital (ref \# 6257). A written and verbal consent was obtained from all participant in the current study.

\section{Consent for publication}

We obtained consent from all the patients included in our study with institutional consent forms.

\section{Availability of data and materials}

The datasets used and/or analyzed during the current study are available from the corresponding author on reasonable request. All data generated or analyzed during this study are included in this published article [and its supplementary information files].

\section{Competing interests}

The authors declare that they have no competing interests. 


\section{Funding}

No funding was received.

\section{Authors' contributions}

All authors carried out and participated in the surgical procedures, MF, IK \& AAB conceived the study, participated in study design and sequence alignment, and drafted the manuscript. ABB, MB \&AB helped to draft and critically revise the manuscript. MF, AB \& IK participated in data collection and performance of the statistical analysis. AAB,MF, MB\&IK participated in study coordination, and critical revision. All authors have read and approved the final manuscript.

\section{Acknowledgments}

We would like to give special thanks, admiration, and respect to all emergency members for their kind help, guidance and valuable support.

\section{References}

1. Stephens PL, Mazzucco JJ. Comparison of ultrasound and the Alvarado score for the diagnosis of acute appendicitis. Conn Med mars. 1999;63(3):137-40.

2. Ilves I, Fagerström A, Herzig K-H, Juvonen P, Miettinen P, Paajanen H. Seasonal variations of acute appendicitis and nonspecific abdominal pain in Finland. World J Gastroenterol WJG. april 2014;14(14):4037-42. 20(.

3. Viniol A, Keunecke C, Biroga T, Stadje R, Dornieden K, Bösner S. m.fl. Studies of the symptom abdominal pain-a systematic review and meta-analysis. Fam Pract oktober. 2014;31(5):517-29.

4. Bhangu A, Søreide K, Di Saverio S, Assarsson JH, Drake FT. Acute appendicitis: modern understanding of pathogenesis, diagnosis, and management. Lancet Lond Engl. september 2015;26(10000):1278-87. 386(.

5. Gomes CA, Abu-Zidan FM, Sartelli M, Coccolini F, Ansaloni L, Baiocchi GL. m.fl. Management of Appendicitis Globally Based on Income of Countries (MAGIC) Study. World J Surg december. 2018;42(12):3903-10.

6. Andersson RE, Hugander AP, Ghazi SH, Ravn H, Offenbartl SK, Nyström PO. m.fl. Why does the clinical diagnosis fail in suspected appendicitis? Eur J Surg Acta Chir oktober. 2000;166(10):796802.

7. Krajewski S. Impact of computed tomography of the abdomen on clinical outcomes in patients with acute right lower quadrant pain: a meta-analysis. Can J Surg 01 februari. 2011;54(1):43-53.

8. Ozao-Choy J, Kim U, Vieux U, Menes TS. Incidental findings on computed tomography scans for acute appendicitis: prevalence, costs, and outcome. Am Surg november. 2011;77(11):1502-9. 
9. Chong CF, Adi MIW, Thien A, Suyoi A, Mackie AJ, Tin AS. m.fl. Development of the RIPASA score: a new appendicitis scoring system for the diagnosis of acute appendicitis. Singapore Med $\mathrm{J}$ mars. 2010;51(3):220-5.

10. Andersson $M$, Andersson RE. The appendicitis inflammatory response score: a tool for the diagnosis of acute appendicitis that outperforms the Alvarado score. World J Surg augusti. 2008;32(8):18439.

11. Damburacı N, Sevinç B, Güner M, Karahan Ö. Comparison of Raja Isteri Pengiran Anak Saleha Appendicitis and modified Alvarado scoring systems in the diagnosis of acute appendicitis. ANZ J Surg april. 2020;90(4):521-4.

12. Drake FT, Mottey NE, Farrokhi ET, Florence MG, Johnson MG, Mock C. m.fl. Time to appendectomy and risk of perforation in acute appendicitis. JAMA Surg augusti. 2014;149(8):837-44.

13. Shuaib A. Evaluation of modifi ed Alvarado scoring system and RIPASA scoring system as diagnostic tools of acute appendicitis. World J Emerg Med. 2017;8(4):276.

14. Alvarado A. A practical score for the early diagnosis of acute appendicitis. Ann Emerg Med maj. 1986;15(5):557-64.

15. Jang SO, Kim BS, Moon DJ. [Application of alvarado score in patients with suspected appendicitis]. Korean J Gastroenterol Taehan Sohwagi Hakhoe Chi juli. 2008;52(1):27-31.

16. Karami MY, Niakan H, Zadebagheri N, Mardani P, Shayan Z, Deilami I. Which One is Better? Comparison of the Acute Inflammatory Response, Raja Isteri Pengiran Anak Saleha Appendicitis and Alvarado Scoring Systems. Ann Coloproctology december. 2017;33(6):227-31.

17. Chong CF, Thien A, Mackie AJ, Tin AS, Tripathi S, Ahmad MA. m.fl. Comparison of RIPASA and Alvarado scores for the diagnosis of acute appendicitis. Singapore Med J maj. 2011;52(5):340-5.

18. Rathod S, Ali I, Bawa AS, Singh G, Mishra S, Nongmaithem M. Evaluation of Raja Isteri Pengiran Anak Saleha Appendicitis score: A new appendicitis scoring system. Med J Dr Patil Univ. 2015;8(6):744.

19. Bolívar-Rodríguez MA, Osuna-Wong BA, Calderón-Alvarado AB, Matus-Rojas J, Dehesa-López E, Peraza-Garay F de. J. [Comparative analysis of diagnostic scales of acute appendicitis: Alvarado, RIPASA and AIR]. Cir Cir. 2018;86(2):169-74.

20. Scott AJ, Mason SE, Arunakirinathan M, Reissis Y, Kinross JM, Smith JJ. Risk stratification by the Appendicitis Inflammatory Response score to guide decision-making in patients with suspected appendicitis. Br J Surg april. 2015;102(5):563-72.

21. de Castro SMM, Ünlü Ç, Steller EPh, van Wagensveld BA, Vrouenraets BC. Evaluation of the Appendicitis Inflammatory Response Score for Patients with Acute Appendicitis. World J Surg juli. 2012;36(7):1540-5.

\section{Figures}




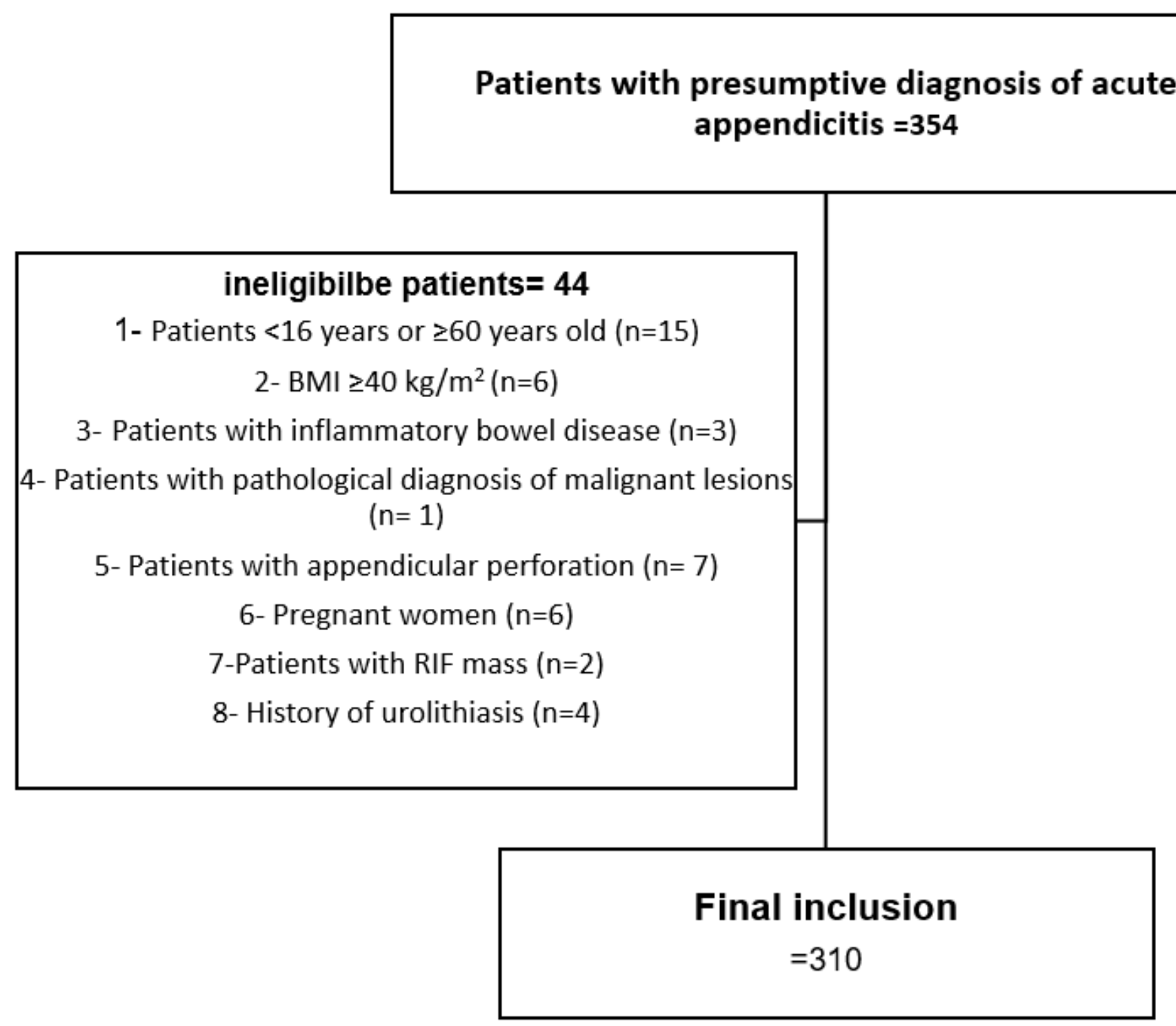

Figure 1

Flow chart of the included patients 


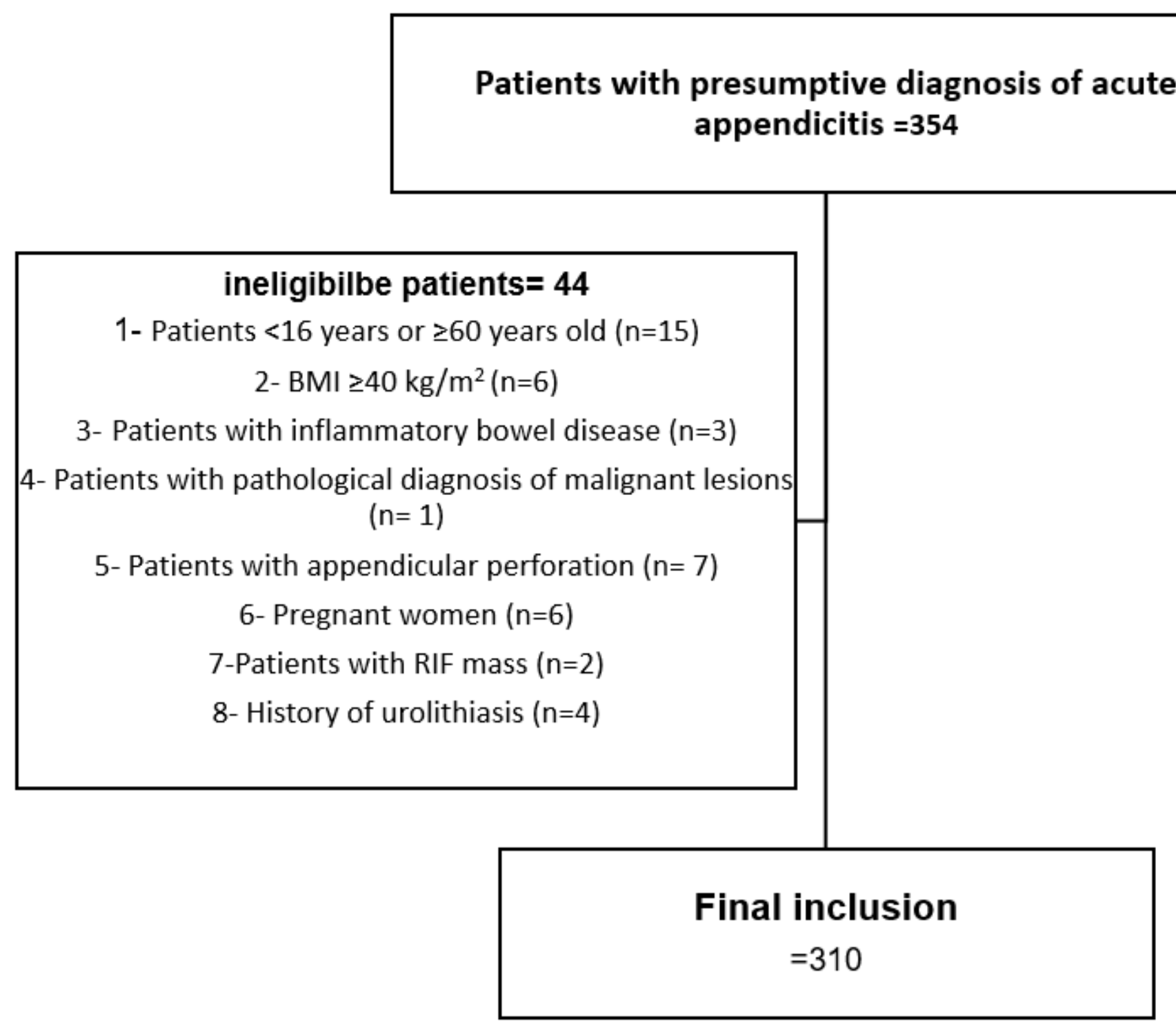

Figure 1

Flow chart of the included patients 


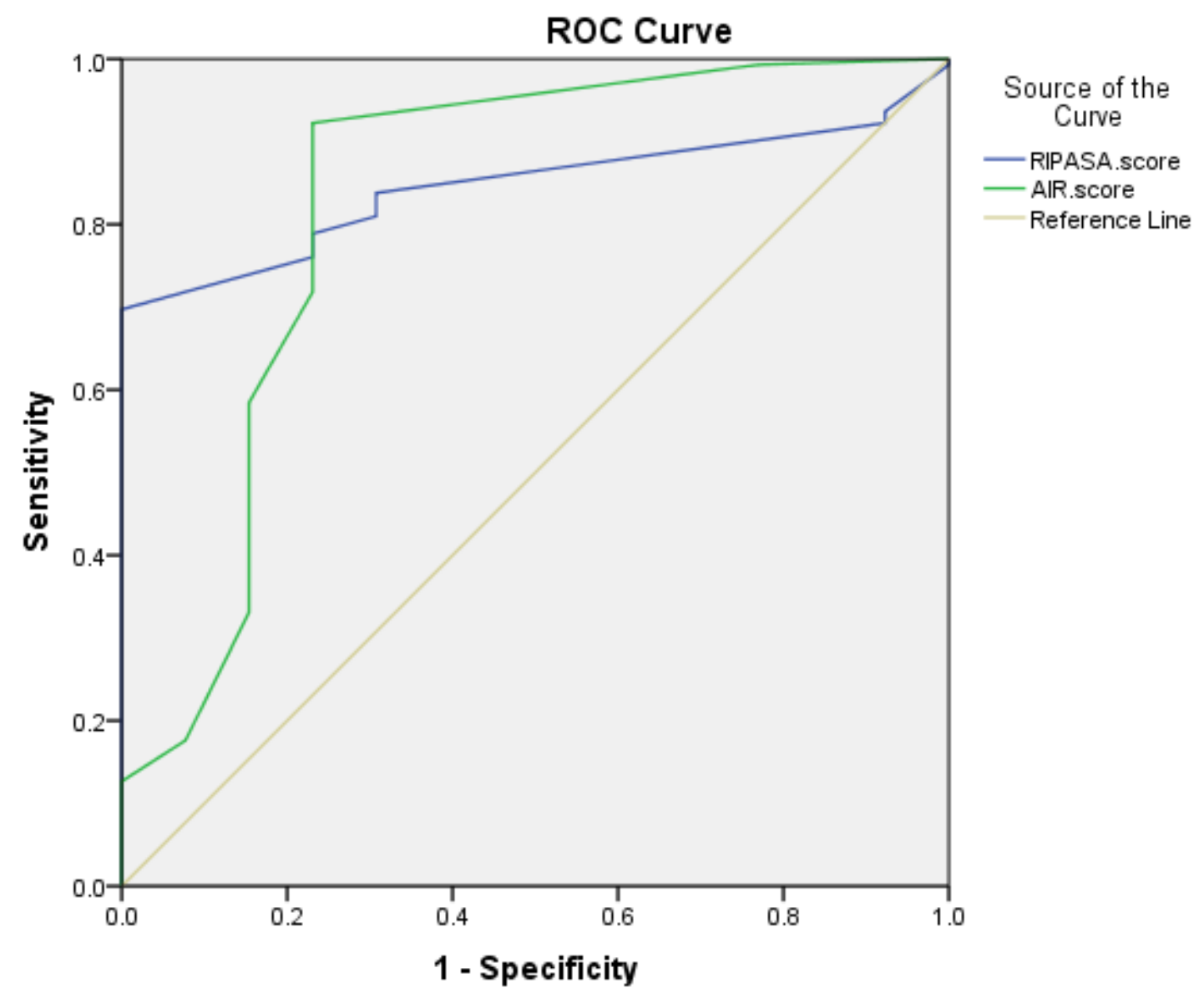

Diagonal segments are produced by ties.

Figure 2

ROC curves obtained by calculating the results of both scores. 


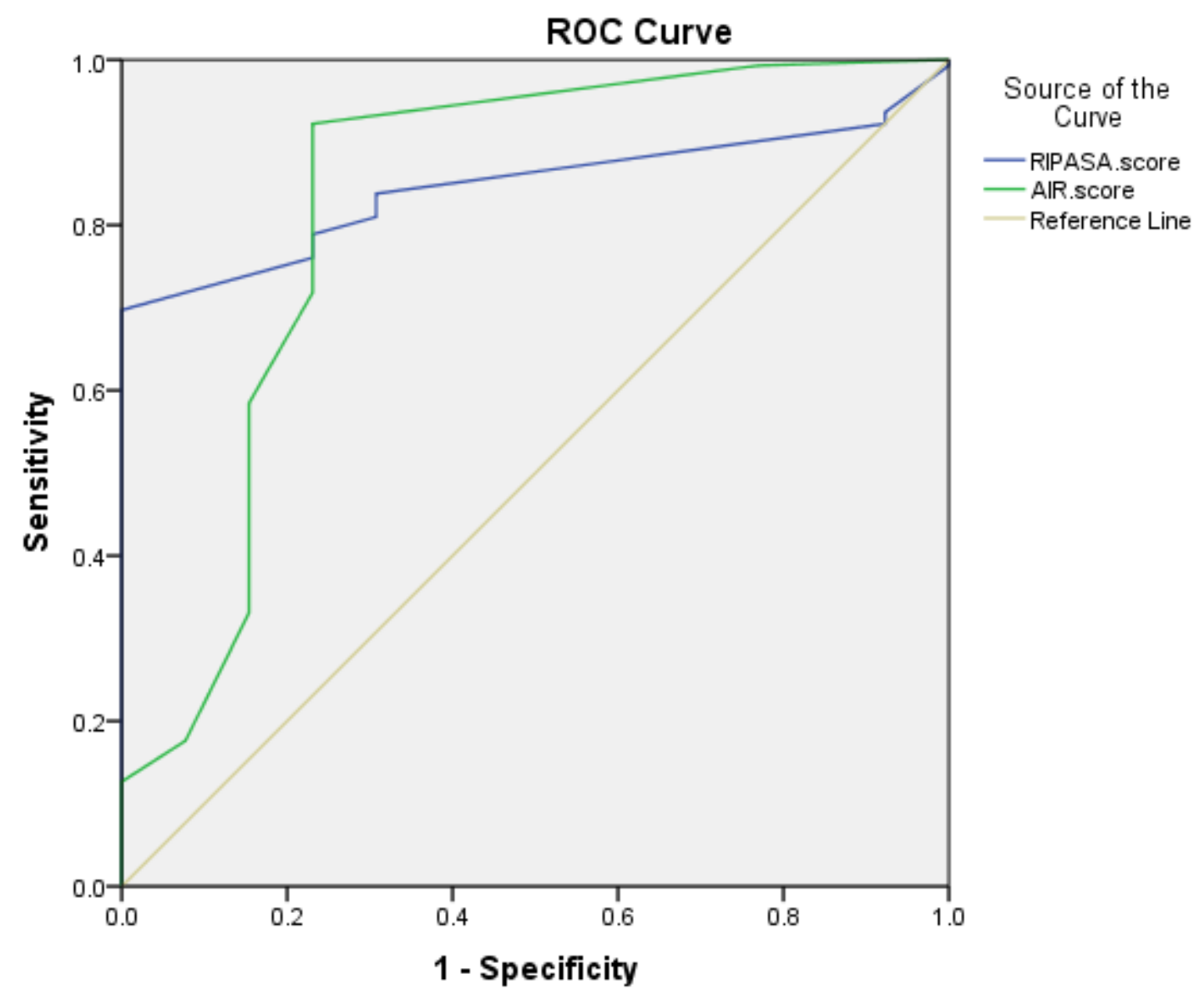

Diagonal segments are produced by ties.

Figure 2

ROC curves obtained by calculating the results of both scores. 


\section{Post-operative complications}

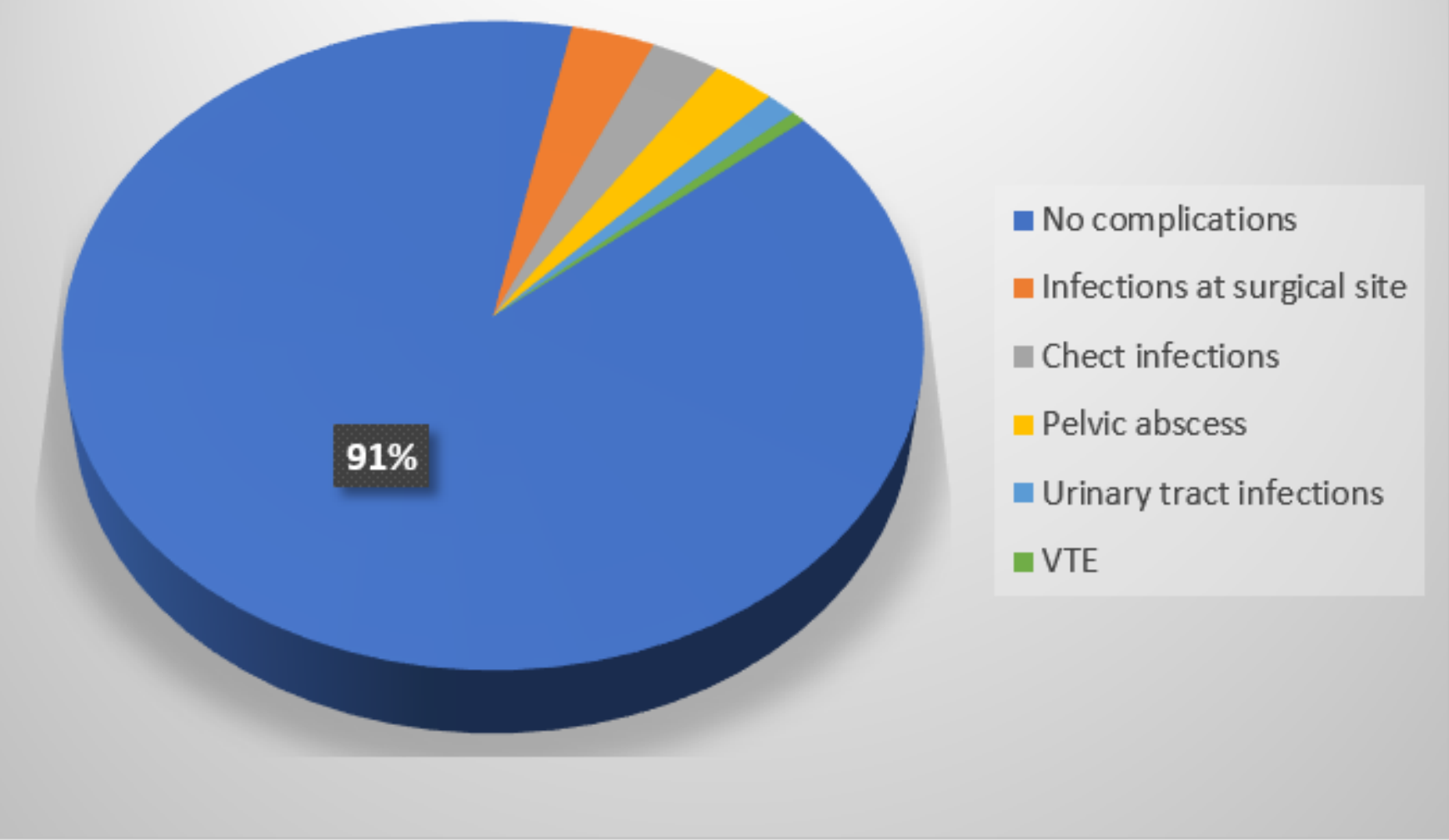

Figure 3

Post-operative complications 


\section{Post-operative complications}

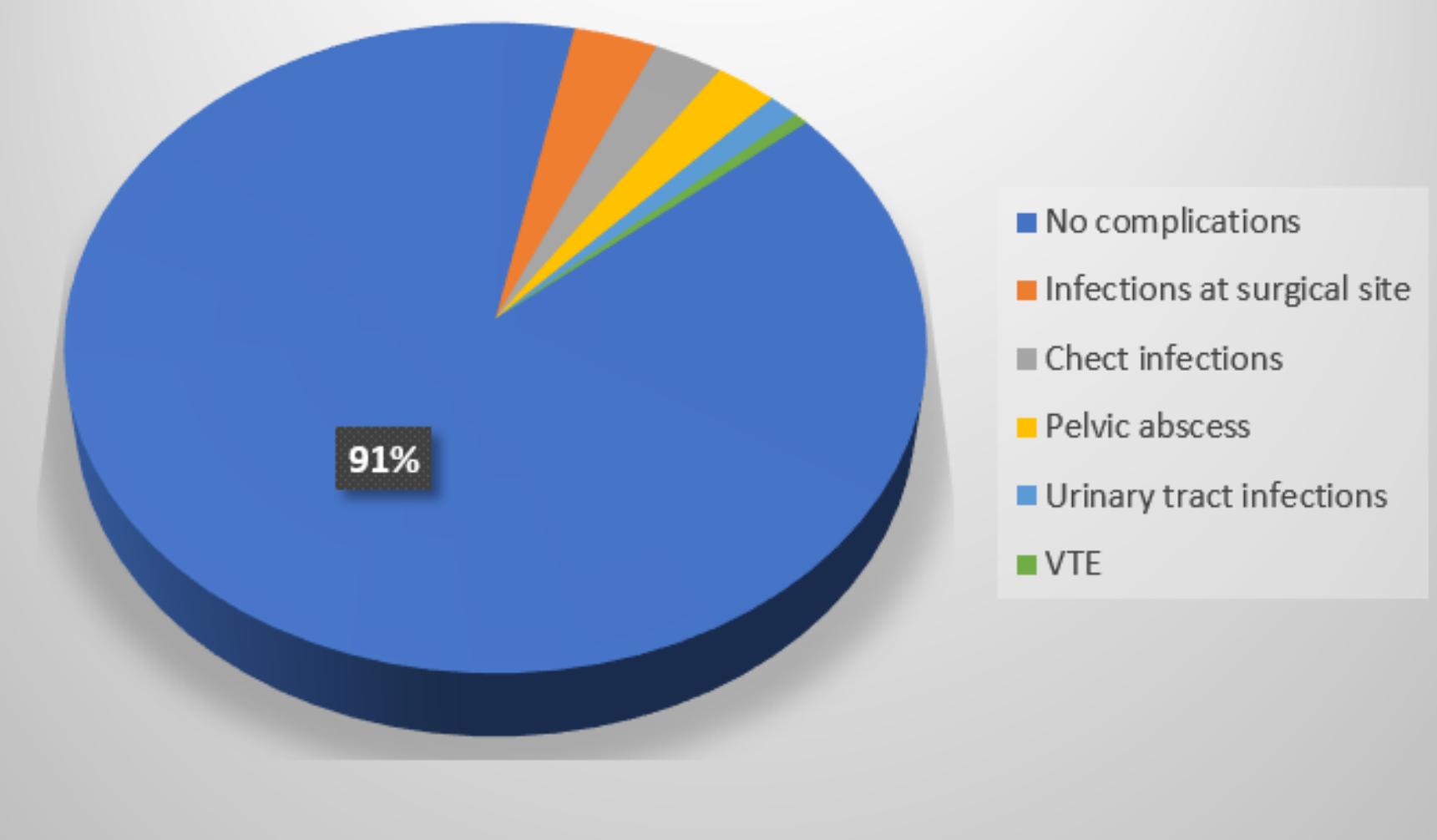

Figure 3

Post-operative complications 